\title{
High-speed camera-based measurement system for aeroacoustic investigations
}

\author{
Johannes Gürtler ${ }^{1}$, Daniel Haufe ${ }^{1}$, Anita Schulz ${ }^{2}$, Friedrich Bake ${ }^{2}$, Lars Enghardt ${ }^{2,3}$, Jürgen Czarske ${ }^{1}$, \\ and Andreas Fischer ${ }^{1}$ \\ ${ }^{1}$ Chair of Measurement and Sensor System Techniques, Department of Electrical Engineering and Information \\ Technology, TU Dresden, Helmholtzstr. 18, 01069 Dresden, Germany \\ ${ }^{2}$ Institute of Propulsion Technology, German Aerospace Center (DLR), 10623 Berlin, Germany \\ ${ }^{3}$ Institute of Fluid Dynamics and Technical Acoustics, TU Berlin, 10623 Berlin, Germany
}

Correspondence to: Johannes Gürtler (johannes.guertler@tu-dresden.de)

Received: 5 July 2015 - Revised: 11 February 2016 - Accepted: 17 March 2016 - Published: 6 April 2016

\begin{abstract}
The interaction of sound and flow enables an efficient noise damping. Inevitable for understanding of this aeroacoustic damping phenomenon is the simultaneous measurement of flow and sound fields. Optical sensor systems have the advantage of non-contact measurements. The necessary simultaneous determination of sound levels and flow velocities with high dynamic range has major hurdles. We present an approach based on frequency-modulated Doppler global velocimetry, where a high-speed CMOS camera with data rates over 160 MSamples s $^{-1}$ of velocity samples is employed. Using the proposed system, two-component flow velocity measurements are performed in a three-dimensional region of interest with a spatial resolution of $224 \mu \mathrm{m}$, based on single-pixel evaluation, and a measurement rate of $10 \mathrm{kHz}$. The sensor system can simultaneously capture sound and turbulent flow velocity oscillations down to a minimal power density of $40.5\left(\mathrm{~mm} \mathrm{~s}^{-1}\right)^{2} \mathrm{~Hz}^{-1}$ in a frequency range up to $5 \mathrm{kHz}$. The presented measurements of the interaction of sound and flow support the hypothesis that the sound energy is transferred into flow energy.
\end{abstract}

\section{Introduction}

\subsection{Motivation}

In order to attenuate the noise of modern jet engines or gas turbines, perforated liners as depicted in Fig. 1 are used (Eldredge and Dowling, 2003). By applying a bias flow through the perforation of such liners it is possible to increase the efficiency and bandwidth of the damping effect (Bechert, 1980). This effect is partially based on the energy transfer from the sound wave into the bias flow as sound-excited oscillations of the flow velocity. In order to be able to design or operate bias flow liners with a high sound damping efficiency, it is desirable to understand the interaction of the sound wave with the flow field. For this reason numerical simulations have been performed (Zhao et al., 2015), but they are often limited because of high calculation efforts, model assumptions or invalid model simplifications and need to be validated. Thus, non-invasive measurement techniques are required that en- able the simultaneous acquisition of the mean flow velocity and the sound-excited oscillation of the velocity in a threedimensional (3-D) region of interest above the liner surface. Furthermore, turbulence spectra of the flow velocity need to be analysed in order to quantify the energy transfer from the sound wave to the flow turbulence that contributes to the sound damping. The maximum damping efficiency of the used liner lies at approximately $1 \mathrm{kHz}$ (Schulz et al., 2015). For this reason, a high measurement rate of $10 \mathrm{kHz}$ is chosen here in order to sample this maximum as well as flow turbulence up to $5 \mathrm{kHz}$ properly, according to the NyquistShannon sampling theorem. Additionally, a short experimental time is needed to reduce environmental influences on the experiment, such as ambient temperature and pressure variations. Hence, a planar (2-D) measurement system is needed in order to measure the entire 3-D region of interest for several minutes. The combination of such imaging systems with the number of measurement points $N_{\mathrm{p}}$ and the required mea- 


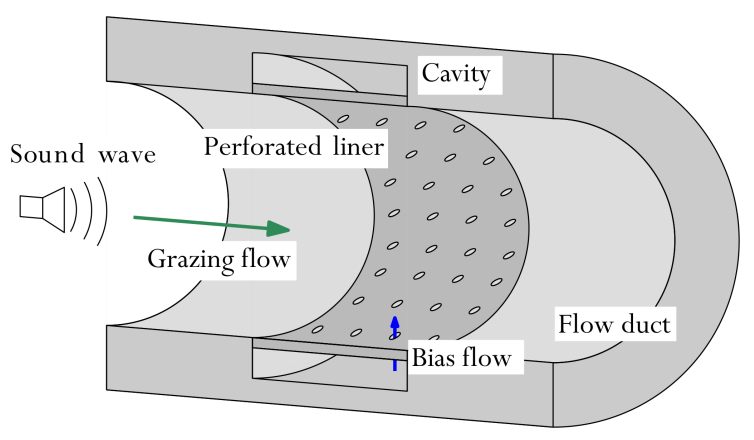

Figure 1. Sketch of a perforated bias flow liner with a cavity behind, installed in a flow duct.

surement rate $f_{\mathrm{s}}$ necessitates a sensor system with high data rates

$f_{\mathrm{d}}=f_{\mathrm{s}} \cdot N_{\mathrm{p}} \cdot N_{\mathrm{c}}$

in the range of MSampless $\mathrm{s}^{-1}$, where $N_{\mathrm{c}}$ is the number of simultaneously measured velocity components. These aforementioned requirements are fulfilled by a high-speed camera-based measurement system.

\subsection{State of the art}

For measurements of small-scale flow velocity fluctuations in the range of several $\mathrm{kHz}$, hot-wire anemometry is a commonly applied technique providing high sensitivity and bandwidth up to $100 \mathrm{kHz}$ (Watmuff, 1995). However, it is a pointwise and invasive technique potentially disturbing the flow and sound field.

Non-invasive, optical measurements of the acoustic particle velocity (APV), i.e. the sound-related particle oscillation velocity (Hann and Greated, 1999; Taylor, 1976) and the flow velocity (Thompson and Atchley, 2005), were realized by using laser Doppler anemometry (LDA). These systems detect the Doppler-shifted frequency of light scattered at single particles moving with the flow with negligible slip and provide data rates in the range of several 10 MSamples s $^{-1}$ (Hann and Greated, 1999). Standard LDA systems yield a point-wise measurement, which objects the requirement of an imaging technique. Enhanced LDA sensor principles with a linear (known as profile sensor) and planar (known as field sensor) measurement volume have been presented (Voigt et al., 2008; Meier and Roesgen, 2012). However, for all LDA principles the measurement rate equals the random particle rate passing the measurement volume observed by one detector element. Hence, a high-speed measurement with a constant measurement and data rate is not possible.

Particle image velocimetry (PIV) offers a simultaneous planar measurement of the flow velocity and the APV (Hann and Greated, 1997; Rupp et al., 2010). It is based on the correlation of interrogation windows, i.e. sections from two subsequently captured images of illuminated particles within a light sheet. Using PIV systems, measurements of the APV were realized with measurement rates up to $20 \mathrm{kHz}$ (Henning et al., 2013). Furthermore, measurements at a bias flow liner were performed with a PIV system phase-synchronous to the acoustic excitation signal (Schulz et al., 2014). However, standard PIV systems provide only two velocity components to be measured simultaneously, while all three velocity components of the complex flow are required. In addition, the desired measurement system needs a dynamic range of the order of $3 \times 10^{3}$ to measure both typical amplitudes of the APV of the order of $10 \mathrm{~mm} \mathrm{~s}^{-1}$ and superimposed flow velocities up to $30 \mathrm{~m} \mathrm{~s}^{-1}$ (Haufe et al., 2013). Since common PIV systems provide a dynamic range of the order of $10^{2}$ (Adrian, 2005), the application of PIV for aeroacoustic studies requires further attention.

A sufficient dynamic range up to $4 \times 10^{3}$ and high measurement rates up to $50 \mathrm{kHz}$ have been achieved recently using Doppler global velocimetry with sinusoidal frequency modulation (FM-DGV) for aeroacoustic investigations at a bias flow liner (Haufe et al., 2012, 2013). Because of the usage of a linear detector array consisting of eight detectors, an extensive traversing effort is needed to measure a volumetric (3-D) region of interest. Moreover, a low data rate of $8 \cdot 50 \mathrm{kHz} \cong 0.4 \mathrm{MSamples}^{-1}$ is achieved. In order to overcome these drawbacks, the existing FM-DGV system was recently enhanced by implementing a high-speed camera for simultaneous light detection, which allows one velocity component to be measured at a data rate of 800 MSamples s $^{-1}$ (Fischer et al., 2014).

Conventional DGV systems combined with high-speed cameras also provide a high dynamic range over $10^{2}$ as well as high measurement rates in the MHz range (Thurow, 2004). However, neither the camera-based FM-DGV nor the DGV has yet been tested for aeroacoustic studies. It is an open question of whether the sensitivity of the camera sensor is still sufficient to resolve small-scale light intensity fluctuations caused by the sound-excited flow velocity oscillations.

In order to further increase the data rate, the simultaneous measurement of more than one velocity component is desired. Simultaneous planar (2-D) measurements of all three velocity components with a single camera were performed using a hybrid DGV and PIV image processing for the same data set (Willert et al., 2006; Wernet, 2004). Using such systems, measurement rates in the lower $\mathrm{Hz}$ range could be achieved. The idea is here to adapt this approach for the highspeed camera-based FM-DGV measurement system, which thus needs to be supplemented by a PIV image processing. It has to be investigated whether such a hybrid FM-DGV-PIV system with a single camera finally allows one to measure all three components of the mean flow field and the soundexcited flow velocity oscillations. 


\subsection{Aim and structure}

A camera-based FM-DGV system using a high-speed camera is demonstrated for volumetric (3-D) field measurements of the flow velocity including the sound-excited oscillations at a bias flow liner. Because of the larger field of view, the higher resolution of the measurement system and the measurement rate of $10 \mathrm{kHz}$, a data rate of $10 \mathrm{MSamples} \mathrm{s}^{-1}$ is achieved according to Eq. (1). Furthermore, the use of the camera allows planar measurements at the bias flow liner, which reduces the traversing effort significantly to one axis. Finally, the high-speed camera offers the perspective of measuring all three velocity components with a single camera by a hybrid FM-DGV-PIV image evaluation.

In Sect. 2 the principle and the setup of the camera-based FM-DGV measurement system are presented. Its validation with respect to aeroacoustic investigations follows in Sect. 3 by performing a measurement of one velocity component at a generic bias flow liner. In addition, the measurement uncertainty is addressed, since a maximum uncertainty of the velocity smaller than $10 \mathrm{~mm} \mathrm{~s}^{-1}$ is needed to resolve the flow velocity oscillations. Using multiple measurements and two different observation directions, the 3-D region of interest is measured for two components of the mean flow velocity and the sound-excited flow oscillations. The measurement results, including the discussion of the acquired turbulence spectra, are presented in Sect. 4. In Sect. 5, the enhancement of the measurement system towards simultaneous three-component measurements with a single camera by using a hybrid FM-DGV-PIV image evaluation is finally discussed as an outlook.

\section{Measurement principle}

The FM-DGV technique is based on evaluating the velocitydependent Doppler shift of the frequency of light scattered at moving particles in the flow (Müller et al., 2007; Fischer et al., 2007). In Fig. 2a and b the principle measurement setup is depicted. The scattering particles, which follow the flow velocity $\boldsymbol{v}_{\mathrm{f}}$ with negligible slip, are illuminated from the direction $i$ by a narrow-band laser light with the centre frequency $f_{\mathrm{c}}$. Light scattered at these particles exhibits a frequency shift by the Doppler frequency

$f_{\mathrm{D}}=\frac{f_{\mathrm{c}}\|\boldsymbol{o}-\boldsymbol{i}\|}{c} \cdot v$, with $v=\frac{(\boldsymbol{o}-\boldsymbol{i})}{\|\boldsymbol{o}-\boldsymbol{i}\|} \cdot \boldsymbol{v}_{\mathrm{f}}$,

where $\boldsymbol{o}$ is the observation direction, $c$ the speed of light and both vectors $\boldsymbol{i}$ and $\boldsymbol{o}$ are unit vectors. Hence, the Doppler frequency is directly proportional to the velocity component $v$ of $\boldsymbol{v}_{\mathrm{f}}$ along the sensitivity vector $\boldsymbol{o}-\boldsymbol{i}$.

Since $\left(f_{\mathrm{c}}\right)$ is $335 \mathrm{THz}$ for the used laser wavelength of $895 \mathrm{~nm}$ and $f_{\mathrm{D}}$ is merely in the $\mathrm{kHz}$ to $\mathrm{MHz}$ range, $f_{\mathrm{D}}$ cannot be measured directly. Therefore, the scattered light is observed through a molecular absorption cell filled with caesium gas. Adequate for the used laser centre frequency $f_{\mathrm{c}}$, caesium provides a steep slope in the light-frequencydependent transmission curve as sketched in Fig. 2c. Thus, the Doppler shift in the frequency of the received light is transformed into a measurable change of the light intensity behind the absorption cell. However, this intensity signal depends not only on the frequency but also on the intensity of the scattered light. In order to eliminate this cross-sensitivity, the laser light frequency is sinusoidally modulated around the absorption minimum of the absorption cell so that

$f_{\text {Laser }}(t)=f_{\mathrm{c}}+f_{\mathrm{h}} \sin \left(2 \pi f_{\mathrm{m}} t\right)$,

where $f_{\mathrm{m}}$ is the frequency and $f_{\mathrm{h}}$ the amplitude of the modulation. As can be seen in Fig. 2c, the resulting transmitted intensity signal contains higher-order harmonics of the modulation frequency depending on the shifted laser centre frequency. Finally, the quotient

$q\left(f_{\mathrm{D}}\right)=\frac{A_{1}\left(f_{\mathrm{D}}\right)}{A_{2}\left(f_{\mathrm{D}}\right)}$

of the amplitudes $A_{1}$ and $A_{2}$ of the first- and second-order harmonics is evaluated, which is independent of the mean scattered light intensity and solely depends on the flow velocity component $v$. After calibrating the system to determine the relationship $v=v(q)$, the desired velocity $v$ can be obtained from the quotient $q$.

\section{Measurement setup and characterization}

\subsection{Measurement object}

Aeroacoustic investigations are realized at a bias flow liner, using the setup shown in Fig. 3. The measurements are performed at the central orifice of $N=53$ orifices. Each one has the diameter $d=2.5 \mathrm{~mm}$ and the distance $s=8.5 \mathrm{~mm}$ to the neighbouring orifices. Above the liner surface, the grazing flow $\boldsymbol{v}_{\mathrm{g}}$ is realized as a suction flow due to a duct fan and is directed in the negative $x$ direction. The mass flow $\dot{m}=5 \mathrm{kgh}^{-1}$ is stabilized with a mass flow controller and corresponds to an average bias flow velocity $v_{\mathrm{b}}=\dot{m} /\left(N \pi\left(\frac{d}{2}\right)^{2} \rho \alpha\right)=7 \mathrm{~ms}^{-1}$, with the air density $\rho=1.2 \mathrm{~kg} \mathrm{~m}^{-3}$ and the empirical jet contraction factor $\alpha=0.61$ (Heuwinkel et al., 2010). The jet contraction factor is defined as the ratio of the cross-section area at the smallest jet diameter (vena contracta) behind the orifice and the area of the orifice. Based on previous measurements at the bias flow liner using the same flow parameters, the Kolmogorov length scale of the flow can be estimated to $20 \mu \mathrm{m}$ (Haufe et al., 2014a). Furthermore the Kolmogorov timescale can be estimated to $24 \mu$ s (Pope, 2000). Note that this estimation is based on the assumption of isotropic turbulence and the $k-\epsilon$ turbulence model.

In order to study the damping phenomenon, a sinusoidal acoustic excitation signal at $f_{\mathrm{ac}}=867 \mathrm{~Hz}$ with a sound pressure level of $120 \mathrm{~dB}$ is applied using a speaker of the type 


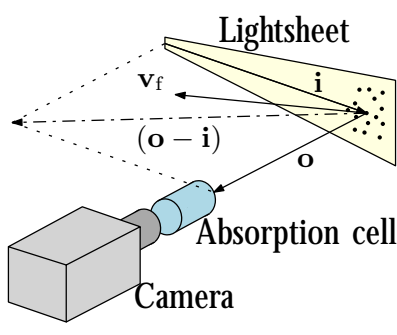

(a)

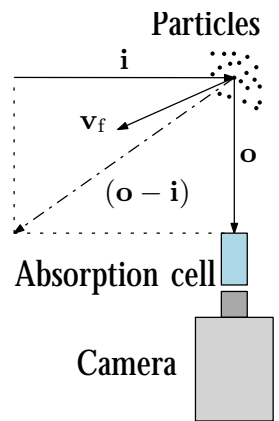

(b)

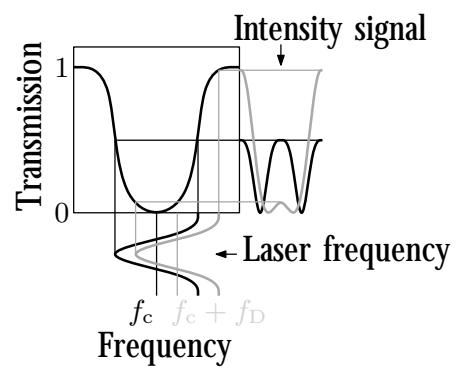

(c)

Figure 2. FM-DGV measurement principle: (a) and (b) the particles are illuminated from the direction $\boldsymbol{i}$, and the scattered light is detected behind a caesium absorption cell. (c) Due to the frequency-dependent transmission curve of the caesium gas, the Doppler frequency shift is transformed into a change of the transmitted light intensity (flow velocity $\boldsymbol{v}_{\mathrm{f}}$, laser centre frequency $f_{\mathrm{c}}$, Doppler frequency $f_{\mathrm{D}}$ ).

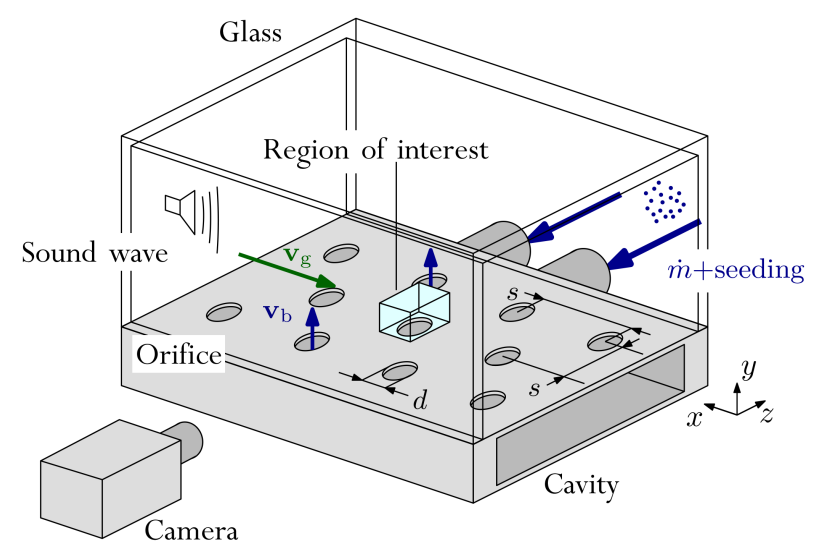

Figure 3. Bias flow liner test rig: a sound wave is excited above the liner facing sheet and is oriented in the direction of the superimposed grazing flow with the velocity $\boldsymbol{v}_{\mathrm{g}}$. Behind the perforated facing sheet is a cavity, in which an additional mass flow $\dot{m}$ is fed that provides the bias flow with the velocity $\boldsymbol{v}_{\mathrm{b}}$ through the perforation of the liner. Note that the point of origin of the used coordinate system is located right in the centre of the orifice.

KU-516 from the manufacturer MONACOR. The total harmonic distortion of the speaker is $0.8 \%$ measured with a flush-mounted microphone.

\subsection{Illumination and detection setup}

The described bias flow liner is expected to exhibit a nonlinear response characteristic, which shall be investigated by analysing the total harmonic distortion of the velocity signal above the liner surface. According to this object, the excitation frequency $f_{\mathrm{ac}}$ and its higher harmonics have to be resolved. Additionally the maximum dissipation of the liner at $1 \mathrm{kHz}$ (Schulz et al., 2015) shall be analysed in the spectrum. Hence, a measurement rate of $10 \mathrm{kHz}$ is necessary in order to properly sample the maximum dissipation range as well as the higher harmonics of the sound-excited oscillations up to
$5 \mathrm{kHz}$, according to the Nyquist-Shannon sampling theorem. For this reason the laser is modulated with $f_{\mathrm{m}}=10 \mathrm{kHz}$, because at least one modulation period is necessary to evaluate the amplitudes $A_{1}$ and $A_{2}$ according to the FM-DGV measurement principle. As a consequence of the chosen modulation frequency and the Nyquist-Shannon sampling theorem, a high camera frame rate over $40 \mathrm{fps}$ is necessary, in order to resolve the second-order harmonic $2 f_{\mathrm{m}}$. Here a camera frame rate of $100 \mathrm{fps}$ is used, which yields 10 camera pictures per modulation period. Such a sequence of 10 subsequent raw data pictures showing the modulation of the detected intensity signal is depicted in Fig. 4.

Since light scattering particles are needed, these are added to the bias flow by a particle generator with four Laskin nozzles and seeded into the measurement volume through the orifices of the liner. The liquid seeding particles are made of diethylhexyl sebacate (DEHS) and have a diameter of about $1 \mu \mathrm{m}$. They are illuminated in the $x-y$ plane by a laser light sheet, which is produced by a power-amplified diode laser. At a wavelength of $895 \mathrm{~nm}$ this laser system provides a maximum of $600 \mathrm{~mW}$ output power, which was used for the measurements. By modulating the laser diode current, the laser frequency is modulated around the centre frequency $f_{\mathrm{c}}$, which is stabilized using a PI controller. Further details of the controlled laser system can be found in Fischer et al. (2013c).

The light scattered at the particles is measured behind the caesium absorption cell with a high-speed camera as shown in Fig. 5a. The cells are temperature stabilized in order to keep the temperature-dependent transmission curve stable. Details of the cell setup and the absorption behaviour of the caesium gas at $895 \mathrm{~nm}$ are given by Fischer et al. (2013c). For imaging a high-speed camera of the type Phantom v1610 from the company Vision Research is used. It provides high frame rates up to $1 \mathrm{Mfps}$ at a frame size of $128 \times 16 \mathrm{px}$, which is reduced to the used frame rate of $100 \mathrm{kfps}$ at a frame size of $128 \times 128 \mathrm{px}$. Based on the single-pixel resolution of the high-speed camera-based FM-DGV, one velocity value per 

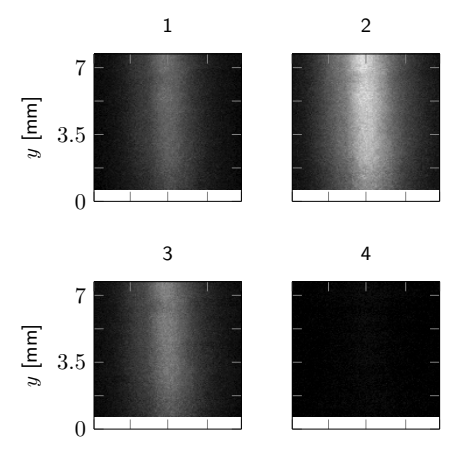

5

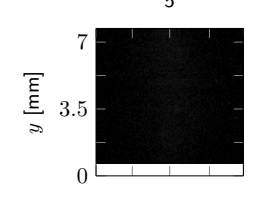

7

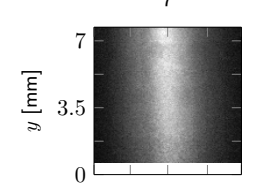

9

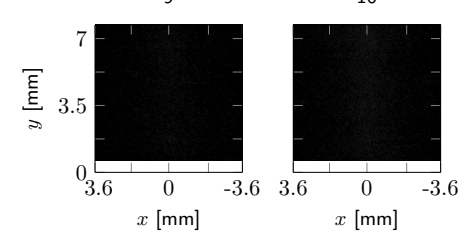

Figure 4. Sequence of 10 subsequent raw pictures, i.e. one modulation period according to $f_{\mathrm{m}}=100 \mathrm{kHz}$. The light intensity is modulated according to the modulation laser frequency (see Fig. 2).

pixel can be measured. This yields a maximum data rate of $128 \cdot 128 \cdot f_{\mathrm{m}} \approx 160 \mathrm{MSamples}^{-1}$ for the proposed setup.

The imaging system, which consists of a Keplerian telescope setup, contains the caesium absorption cell between the two lenses and provides a magnification $M=1: 2$. The observed region spans $7.2 \times 7.2 \mathrm{~mm}^{2}$ in the $x-y$ plane and is located above the central orifice of the liner beginning at $y>600 \mu \mathrm{m}$ in order to avoid the detection of reflexes from the liner surface. The volumetric (3-D) velocity field is finally obtained by traversing the light sheet together with the camera along the $z$ direction in eight steps of $450 \mu \mathrm{m}$, which is depicted in Fig. 5b. For averaging, five repeated acquisitions, each with a duration of $18 \mathrm{~s}$ (i.e. $1.8 \times 10^{5}$ frames), are recorded and evaluated for each $z$ position. Note that the point of origin of the used coordinate system is located right in the centre of the orifice.

\subsection{Data evaluation}

In principle, the FM-DGV technique combined with a camera allows a high spatial resolution up to the size of a single
Table 1. Specifications of the measurement system including the laser and the high-speed camera.

\begin{tabular}{lr}
\hline Max. laser power & $600 \mathrm{~mW}$ \\
Light-sheet thickness & $300 \mu \mathrm{m}$ \\
Modulation rate $f_{\mathrm{m}}$ & $10 \mathrm{kHz}$ \\
Pixel size & $28 \times 28 \mu \mathrm{m}^{2}$ \\
Camera resolution & $128 \times 128 \mathrm{px}$ \\
Binned resolution & $34 \times 34 \mathrm{px}$ \\
Traversing & $8 \times 450 \mu \mathrm{m}$ \\
Magnification $M$ & $1: 2$ \\
Measurement volume & $7.2 \times 7.2 \times 3.6 \mathrm{~mm}^{3}$ \\
Spatial resolution & $224 \times 224 \times 300 \mu \mathrm{m}^{3}$ \\
\hline
\end{tabular}

pixel. However, a binning of $4 \times 4$ pixel is applied here to reduce the influence of fluctuations of the scattered light due to spatial averaging (Fischer et al., 2008). The use of this binning reduces the data rate to $10 \mathrm{MSamples} \mathrm{s}^{-1}$ and yields a spatial resolution of $224 \times 224 \times 300 \mu \mathrm{m}^{3}$. Since its dimension in each direction is about 1 order of magnitude smaller than the orifice diameter, the spatial resolution is sufficient.

With the presented setup it is possible to measure one velocity component. According to Eq. (2), the direction of the measured velocity component $v_{1}$ is along the vector $\left(\boldsymbol{o}_{1}-\boldsymbol{i}\right)$ as sketched in Fig. 5b. In the Sect. 3.4 the results of the one-component measurement setup are used for validation and uncertainty analysis. Since a one-component measurement is not sufficient to understand the flow behaviour at the bias flow liner, a two-component measurement is also performed by adding a second measurement at the same positions but with an anti-parallel observation direction $\boldsymbol{o}_{2}=-\boldsymbol{o}_{1}=(0,0,1)$, which enables the measurement of the velocity component $v_{2}$. Through the coordinate transformation

$\left(\begin{array}{l}v_{y} \\ v_{z}\end{array}\right)=\left(\begin{array}{cc}\cos \varphi & -\sin \varphi \\ \sin \varphi & \cos \varphi\end{array}\right)\left(\begin{array}{l}v_{1} \\ v_{2}\end{array}\right)$ with $\varphi=45^{\circ}$,

the measured velocity components $v_{1}$ and $v_{2}$ are thereby transformed into the Cartesian components $v_{y}$ in $y$ direction and $v_{z}$ in $z$ direction (Fischer et al., 2013b). The results of the two-component measurement are presented in Sect. 4. An overview of the measurement system specifications is given in Table 1.

\subsection{Characterization measurement}

In order to validate the capability of the measurement system for aeroacoustic investigations at a bias flow liner, the system is examined with respect to two requirements. First, the measurement system has to be able to resolve soundexcited oscillations of the flow velocity. At the same time, it has to allow the detection of flow turbulence down to $10^{-4}\left(\mathrm{~m} \mathrm{~s}^{-1}\right)^{2} \mathrm{~Hz}^{-1}$ in the case of flow velocities up to $30 \mathrm{~m} \mathrm{~s}^{-1}$ (Haufe et al., 2013). Thus, it also has to provide 


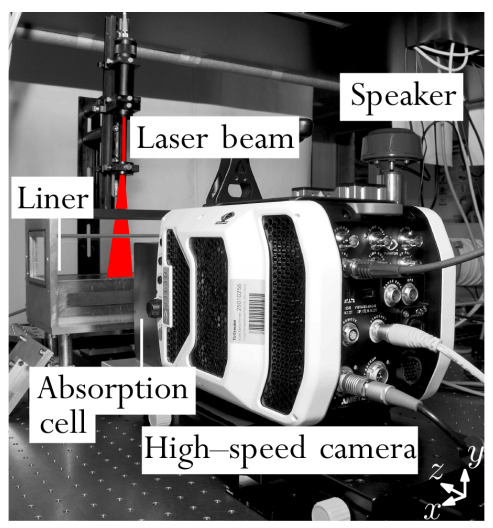

(a)

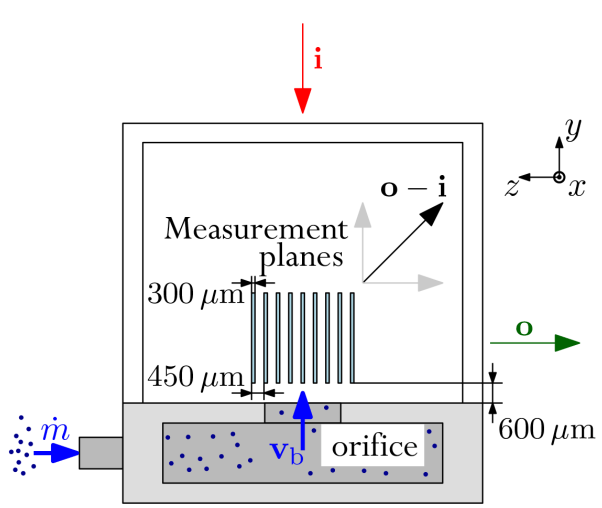

(b)

Figure 5. Measurement setup: (a) the test rig is acoustically excited by a speaker, and the sound-flow interaction at the liner is measured using the FM-DGV system in combination with a high-speed camera. The suction unit for generating the grazing flow $\boldsymbol{v}_{\mathrm{g}}$ is installed behind the speaker. (b) The laser light sheet and the imaging system are traversed in $z$ direction in steps of $450 \mu \mathrm{m}$. In the nine resulting measurement planes, the velocity component $v$ in the direction of the vector $(\boldsymbol{o}-\boldsymbol{i})$ is measured. Note that the sketch is not drawn to scale in order to gain a better clarity.

a small standard deviation of the measured velocity smaller than $10 \mathrm{~mm} \mathrm{~s}^{-1}$, assuming the used measurement time of $5 \times 18 \mathrm{~s}$.

Due to the known sound excitation frequency $f_{\mathrm{ac}}=867 \mathrm{~Hz}$ the fulfilment of the first requirement can be validated by examining the spectrum of the velocity with respect to flow velocity oscillations at $f_{\text {ac }}$ and its higher harmonics up to $5 f_{\text {ac }}$. Therefore the averaged spectrum at the measurement point at $(x, y, z)=(0,3.6,0 \mathrm{~mm})$ is shown in Fig. 6a as an example, which is calculated using a fast Fourier transformation (FFT) of $5 \times 180000$ velocity samples corresponding to a measurement time of $90 \mathrm{~s}$. The spectrum shows the expected characteristic peaks at $f_{\mathrm{ac}}$ and its higher harmonics up to $5 f_{\text {ac }}$, which validates the capability of the measurement system to detect soundexcited oscillations of the flow velocity. Furthermore, the absolute value in the spectrum decreases with increasing frequency. This is typical for flow turbulence indicating the decay of the vortices according to Kolmogorov's theory and is different from the white noise due to the measurement uncertainty (Fischer et al., 2013a). For frequencies over $3 \mathrm{kHz}$ the spectrum converges towards a constant value $\quad \sqrt{\operatorname{mean}\left(|\mathrm{FFT}(v)|^{2}\right)}=\sigma / \sqrt{900000}=1.5 \mathrm{~mm} \mathrm{~s}^{-1}$, which is a measure of the velocity standard deviation after averaging over 900000 samples, i.e. $90 \mathrm{~s}$. As a result, flow velocity fluctuations down to a power density of $\left(1.5 \mathrm{~mm} \mathrm{~s}^{-1}\right)^{2} \cdot 18 \mathrm{~s}=40.5\left(\mathrm{~mm} \mathrm{~s}^{-1}\right)^{2} \mathrm{~Hz}^{-1}$ (based on a window size of $18 \mathrm{~s}$ ) are successfully resolved in the frequency range up to $5 \mathrm{kHz}$. Note that $\sigma$ denotes the standard deviation of all velocity samples in case of no averaging.

The directly calculated standard deviation $\sigma_{\mathrm{v}}$ of the mean flow velocity from the velocity series is shown in Fig. 6b versus the averaging time $N / f_{\mathrm{m}}$ with $N$ as number of averaged samples. Further the estimated standard deviation $\sigma / \sqrt{N}$ for the assumption of white noise is shown, too. In comparison $\sigma_{\mathrm{v}}$ decreases less with increasing averaging time than $\sigma / \sqrt{N}$. This difference is a result of the flow turbulence (see Fig. 6a), which causes non-white noise. However, for $N>40000$ samples $\sigma_{\mathrm{v}}$ achieves the required uncertainty range $<10 \mathrm{~mm} \mathrm{~s}^{-1}$. The respective theoretical uncertainty limit for the measurement system is $0.3 \mathrm{~mm} \mathrm{~s}^{-1}$ according to Fischer et al. (2013c). As follows, the measured value of $1.5 \mathrm{~mm} \mathrm{~s}^{-1}$ is 1 order of magnitude larger than the theoretical limit. This is expected, since the measured velocity standard deviation contains contributions from the flow turbulence, which is not a measurement uncertainty. Furthermore the uncertainty limit is based on a FM-DGV system with a higher modulation frequency of $100 \mathrm{kHz}$ and an avalanche photodiode array as photodetection unit. The uncertainty is increased by fluctuations of the scattered light intensity, i.e. when the scattered light significantly changes during one modulation period of the FM-DGV system. Accordingly, a high modulation frequency (high temporal resolution) and a spatial averaging (low spatial resolution) enable a low uncertainty due to the fluctuations of the scattered light intensity (Fischer et al., 2007). For this reason it is assumed that the higher standard deviation of the camera-based system results from fluctuations of the scattered light, whose influence is increased due the lower modulation frequency of $10 \mathrm{kHz}$ and the higher spatial resolution. This has to be investigated further in future studies and promises possible system improvements with an optimization of the measurement setup. However, the achieved velocity standard deviation is small enough to fulfil both aforementioned requirements.

Based on the spectrum the total harmonic distortion of the sound-excited periodic flow velocity oscillations is cal- 


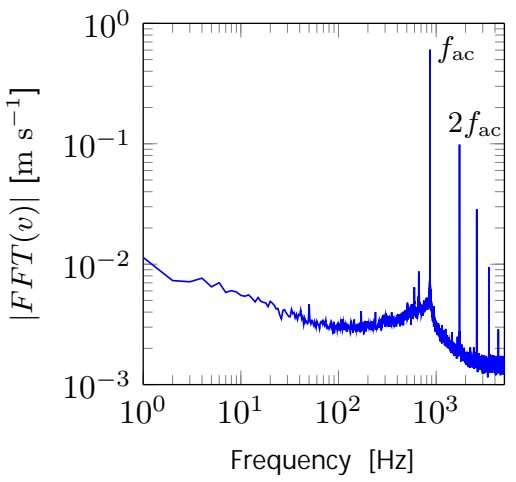

(a)

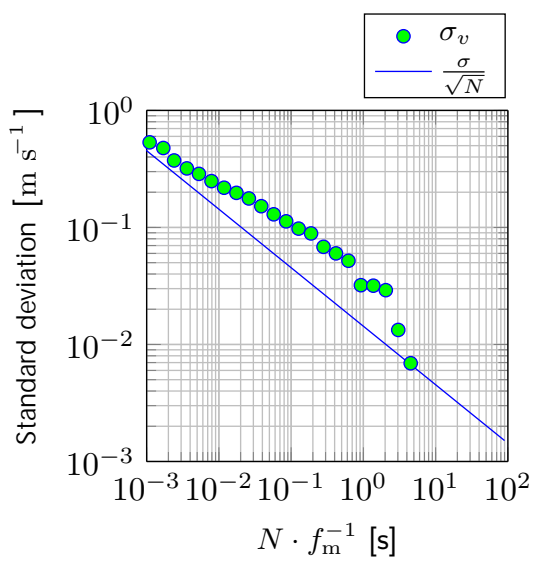

(b)

Figure 6. (a) Spectrum of the flow velocity and (b) standard deviation $\sigma_{\mathrm{v}}$ of the mean flow velocity, calculated directly from the velocity series by averaging over $N$ velocity samples. Estimated standard deviation $\sigma / \sqrt{N}$ for assumption of white noise, calculated using all measured velocity samples without averaging. Both standard deviations depend on the averaging time $N / f_{\mathrm{m}}$ with the measurement rate $f_{\mathrm{m}}$ for the measurement point at $(x, y, z)=(0,3.6,0 \mathrm{~mm})$.

culated to $3 \%$. Hence, the calculated result is larger than the measured total harmonic distortion of $0.8 \%$ of the speaker. Consequently the transfer of the oscillations from the sound wave to the flow velocity has a non-linear response characteristic, as expected from the Navier-Stokes equations being non-linear. Thus, the sound energy is transferred into kinetic energy in the form of flow vortices, which contributes to the damping of the sound like observed by Haufe et al. (2014b) and Schulz et al. (2015). In summary the calculation of the total harmonic distortion also proves the capability of the measurement system to resolve the interaction between sound excitation and flow turbulence. As a result the system is validated to be applicable for aeroacoustic investigations at the bias flow liner.

\section{Aeroacoustic measurements}

The results of the aeroacoustic investigations are presented by evaluating the data of the two-component measurements. First, in order to investigate the mean flow behaviour, an overview of the mean flow velocities in $y$ and $z$ direction is given in Fig. 7. A more detailed view is given in Figs. 8 and 9. The averaging time is $90 \mathrm{~s}$. Note that in order to improve the visibility of the measurement results the $z$ axis is stretched in Fig. 7. The dissipation rate, i.e. the reduction of the sound wave energy at the used bias flow liner operated with the described parameters, is measured using a microphone and amounts to 0.4 of the insert sound energy.

The maximum flow velocity amounts to $\bar{v}_{y}=7.2 \mathrm{~m} \mathrm{~s}^{-1}$ in $y$ direction and $\bar{v}_{z}=3.5 \mathrm{~m} \mathrm{~s}^{-1}$ in $z$ direction, respectively. The magnitude of the velocity $v_{y}$ is in good agreement with the estimated value of $v_{\mathrm{b}}=7 \mathrm{~ms}^{-1}$, calculated from the controlled mass flow $\dot{m}$. Based on the suction flow $\boldsymbol{v}_{\mathrm{g}}$, the mean

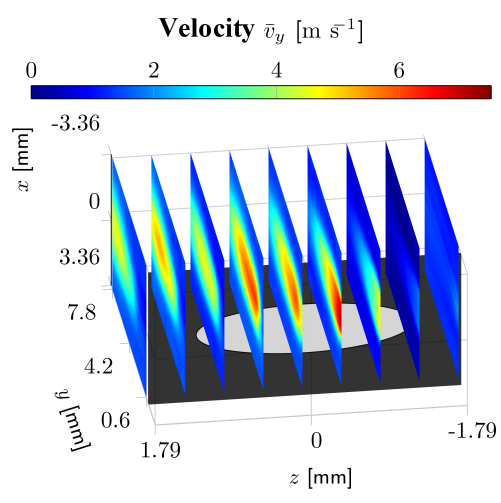

(a)

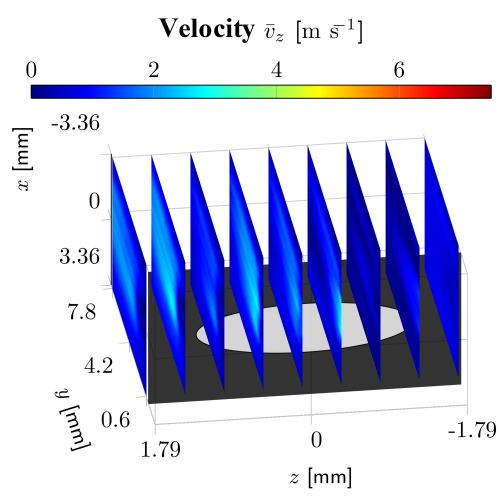

(b)

Figure 7. Mean flow velocity (a) $\bar{v}_{y}$ in $y$ direction and (b) $\bar{v}_{z}$ in $z$ direction.

flow is slightly tilted towards the negative $x$ direction. Furthermore, the flow leaves the orifice slightly oblique in $z$ direction. This is most likely attributed to the asymmetric feed- 


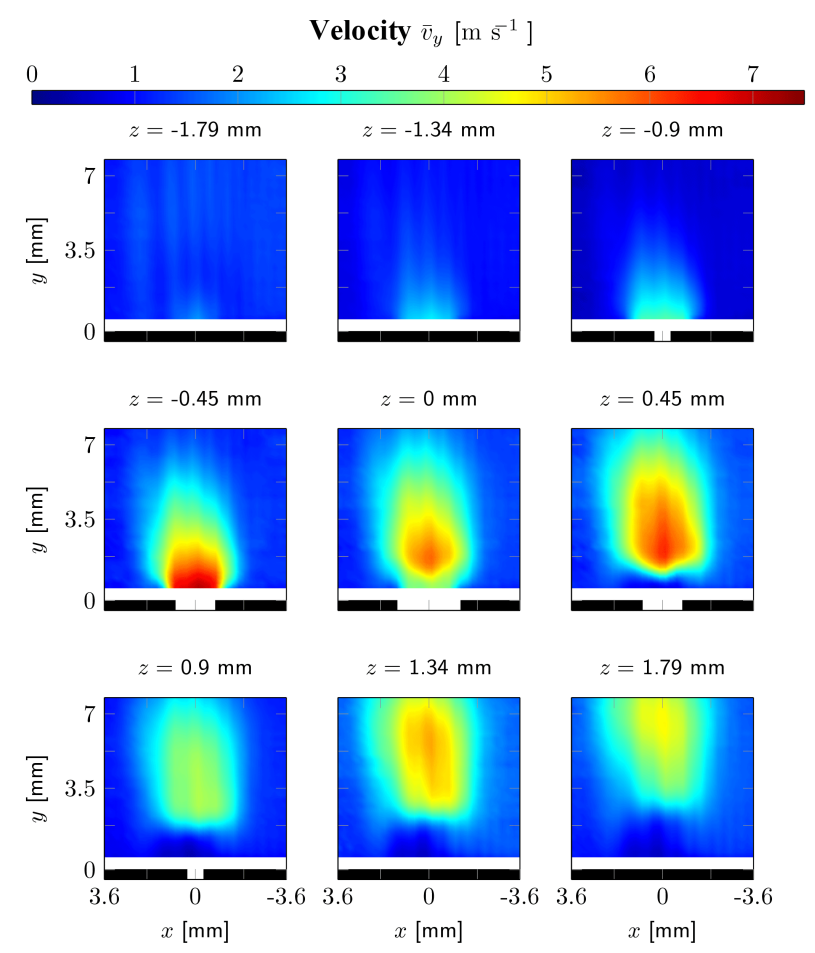

Figure 8. Mean flow velocity $\bar{v}_{y}$ in $y$ direction.

ing of the bias flow only from the positive $z$ direction and the reflection of the flow at the inner wall of the liner cavity. Because of this, the distance of the velocity maximum to the liner surface increases for each measurement plane with increasing $z$.

The existence of sound-excited flow oscillations at a single measurement point was already shown in Sect. 3.4 and will be discussed here further with respect to their spatial and temporal behaviour in the 3-D measurement volume. Such investigations of the excited oscillations are possible by using a phase-resolved analysis of the flow oscillation for the known sound signal with the period $f_{\mathrm{ac}}^{-1}$. The phaseaveraged velocities in the measurement plane at $z=0 \mathrm{~mm}$ are shown in Fig. 10 for the phase angle $\varphi_{\mathrm{ac}}=0 \ldots 2 \pi$ of the acoustic excitation signal. Therefore the velocity samples are divided into 16 groups of the same acoustic phase angle, according to a phase resolution of $\pi / 8$. Then the samples of each group are averaged. As a result, two maxima occur, whose distance of $0.4 \mathrm{~cm}$ is 2 orders of magnitude smaller than the acoustic wavelength of $38 \mathrm{~cm}$. Hence, the measured velocity oscillations are dominated by flow oscillations resulting from vortices. The amplitudes of the sound-excited oscillation velocity are presented in Fig. 11 for both velocity components. A more detailed view of both velocities is shown in Figs. 12 and 13. The calculation of the amplitudes is based on the Fourier coefficient of the time series at $f_{\text {ac }}$. The maximum oscillation amplitude in $y$ direction amounts to $3.8 \mathrm{~m} \mathrm{~s}^{-1}$. Accordingly, it is more than 1 order of mag-

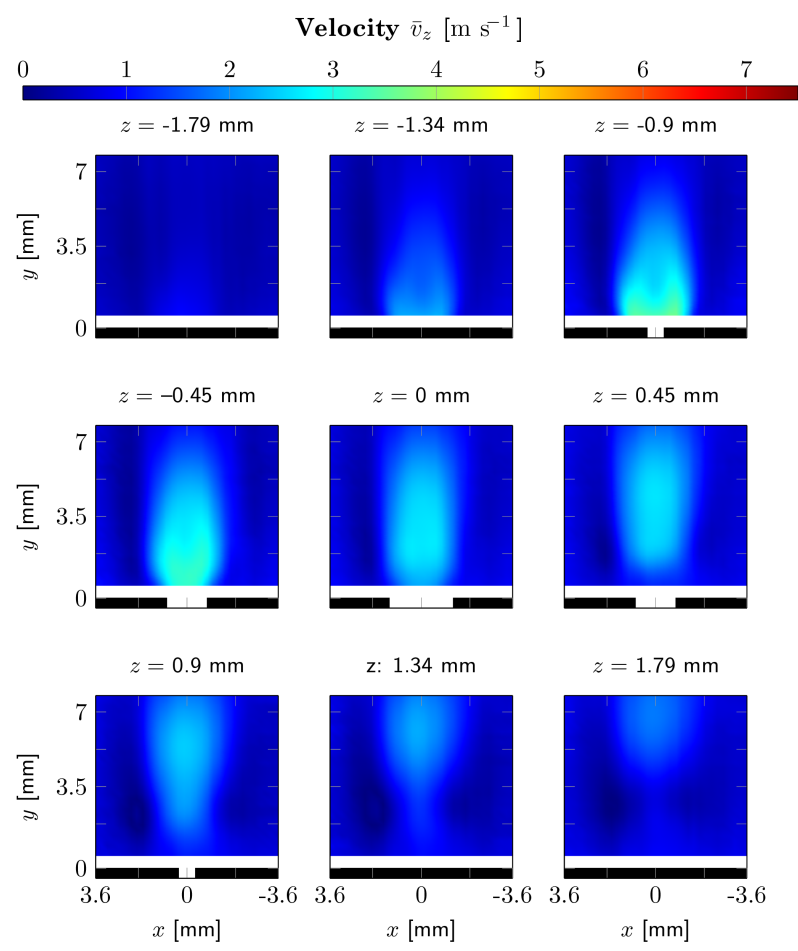

Figure 9. Mean flow velocity $\bar{v}_{z}$ in $z$ direction.

nitude larger than the amplitude of the APV of $48 \mathrm{~mm} \mathrm{~s}^{-1}$. Note that the given amplitude of the APV is based on the used sound pressure level of $120 \mathrm{~dB}$, a temperature of $20^{\circ} \mathrm{C}$ and the assumption of a plane wave. Consequently, the detected oscillations of the flow velocity are mainly sound-excited and prove the interaction of sound and flow velocity.

Like detected for the mean flow velocity field, regions with a large oscillation amplitude exhibit an increasing distance to the liner surface in positive $z$ direction. Also, soundexcited oscillations are located in the vicinity of the orifice rim, which is a region of high vorticity (Heuwinkel et al., 2010). Thus, an enhancement of the mean flow velocity and the vorticity coincide with an enhancement of the energy transfer from the sound into the flow. Furthermore regions of a high vorticity such as the orifice rim exhibit also a large velocity oscillation amplitude. This coincidence implies the generation of vortices fluctuating with the acoustic excitation frequency. According to the time evolution during one acoustic period in Fig. 10, the vortices detach from the liner surface and decompose continuously with increasing distance to the liner surface. The decomposition into smaller vortices at higher frequencies can also be seen in the velocity spectrum in Fig. 6a due to the decreasing value in the spectrum. This behaviour implies further the proceeding decomposition of the vortices, according to Kolmogorov's theory, and their final dissipation into heat. Hence it is shown that the sound damping performance of the bias flow liner is partially based on these sound-exited flow vortices and their dissipation. 

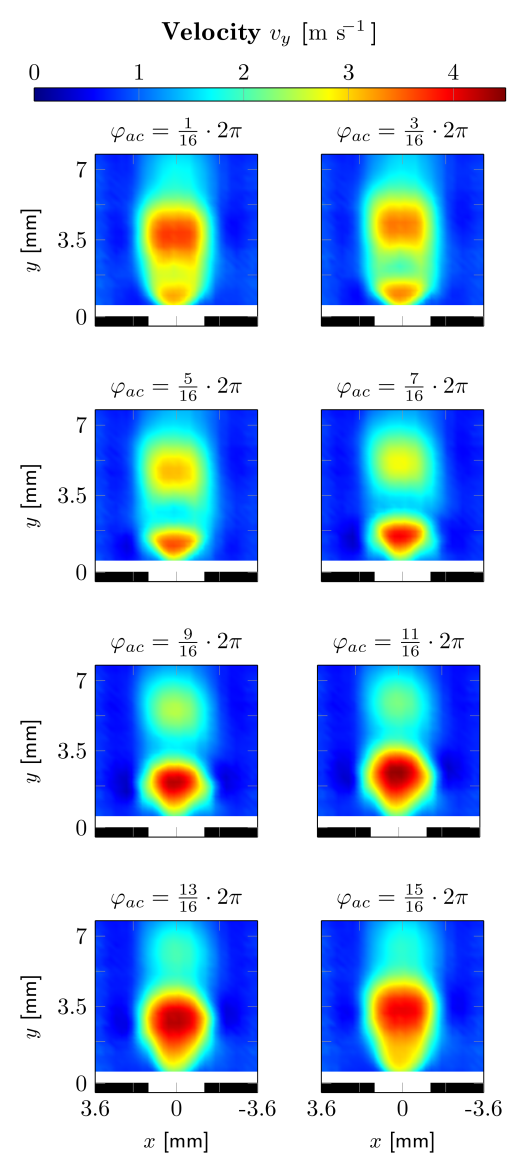

Figure 10. Phase-resolved oscillation of the flow velocity $v_{y}$ at $z=0 \mathrm{~mm}$ for different phase angles $\varphi_{\mathrm{ac}}$ of the sound signal.

\section{Simultaneous three-component measurements with a single camera}

In order to understand the complex flow behaviour at the bias flow liner with respect to flow turbulence, an enhancement of the camera-based FM-DGV system is needed, which enables a simultaneous measurement of all three flow velocity components in one measurement plane. Thus, PIV algorithms are additionally applied to determine the in-plane velocity components. This allows a simultaneous three-component measurement of a planar velocity field using a single camera and, thus, increases the data rate when measuring in the 3-D region of interest. Due to this 3-D three-component measurement, the decomposition of the measured superposition of velocity signals resulting from APV and flow velocity oscillations using the Helmholtz-Hodge decomposition could be performed (Petronetto et al., 2010). However, here only a planar measurement at the position $z=0$ is presented as an example.

The PIV evaluation of the full-resolution images spanning $128 \times 128 \mathrm{px}$ is performed using the open-source software PIVlab. The high seeding concentration, needed for FM-DGV, prohibits a resolution of single particles preferably

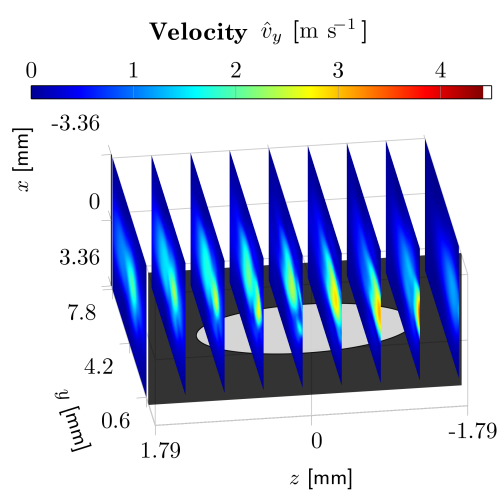

(a)

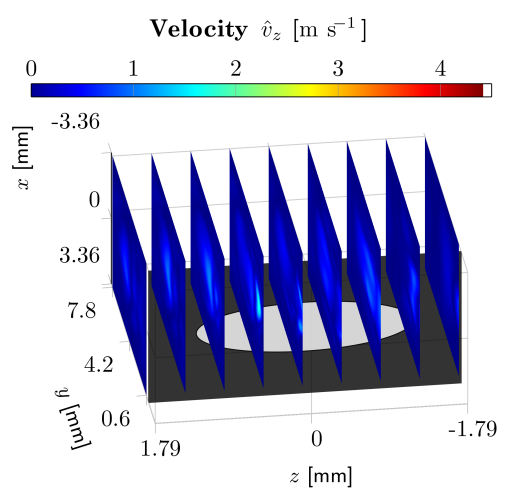

(b)

Figure 11. Amplitude of the sound-excited flow velocity oscillations (a) in $y$ direction and (b) in $z$ direction.

used for PIV. However, since the PIV algorithms calculate the cross-correlation function of shifted interrogation windows it is merely needed to resolve moving structures with a sufficient image contrast. The size of the interrogation windows is decreased over three passes in order to increase the precision and the spatial resolution of the PIV results. In the first pass of the evaluation routine the window size is chosen to $35 \times 35 \mathrm{px}$ and then is decreased over two more passes to $15 \times 15 \mathrm{px}$ and $10 \times 10 \mathrm{px}$. The interrogation window overlap is in all cases adjusted to $50 \%$, which corresponds to a grid of $5 \times 5 \mathrm{px}$ in the third pass of the evaluation routine. This finally results in a spatial resolution of $280 \times 280 \times 300 \mu \mathrm{m}^{3}$ according to the measurement setup in Sect. 3. Due to the symmetric modulation of the laser light frequency nearly around the minimum of the non-linear transmission curve of the caesium, the intensity signal is dominated by the second harmonic at $2 f_{\mathrm{m}}$ as sketched in Fig. 2c. Based on that, two bright images per modulation period with maximum intensity are present (see Fig. 4). These images can be used for the PIV evaluation, obtaining one measurement result per modulation period. Consequently, the PIV measurement rate equals the measurement rate of the FM-DGV and amounts to $10 \mathrm{kHz}$. 


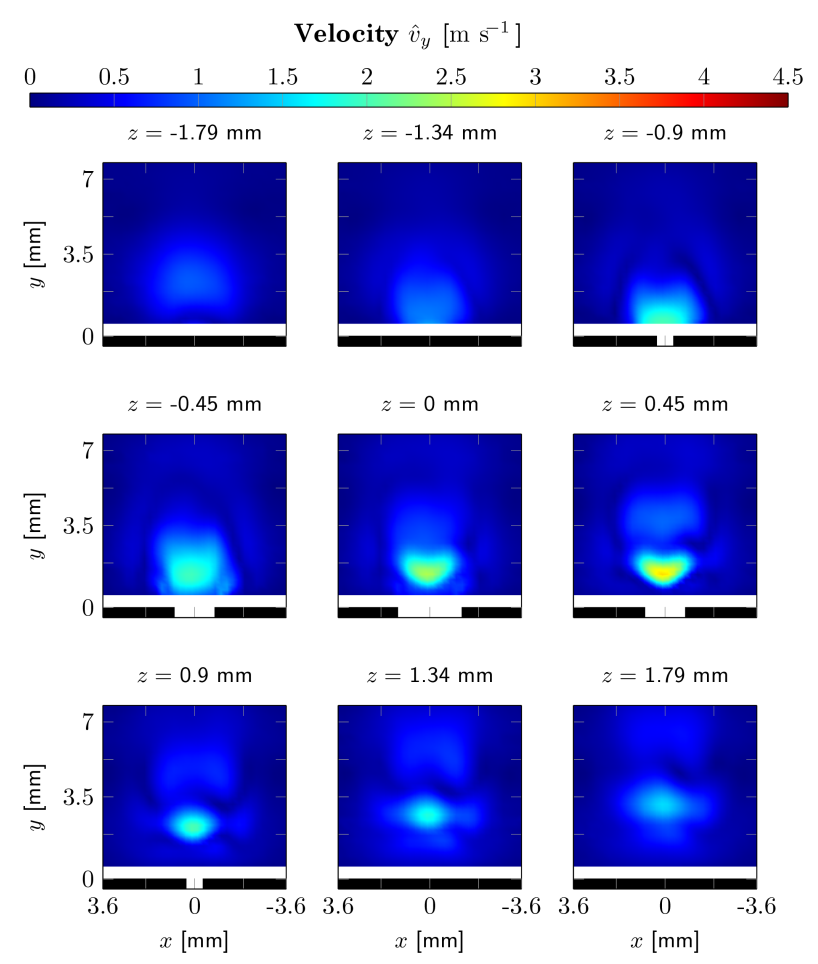

Figure 12. Amplitude of the sound-excited flow velocity oscillations in $y$ direction.

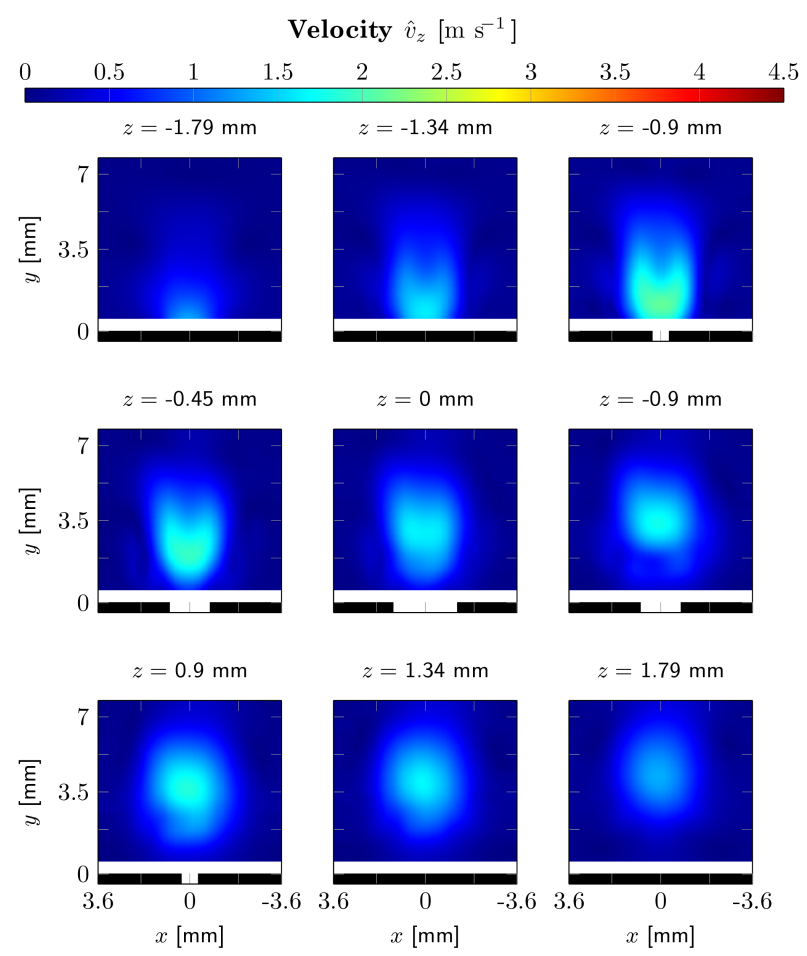

Figure 13. Amplitude of the sound-excited flow velocity oscillations in $z$ direction.

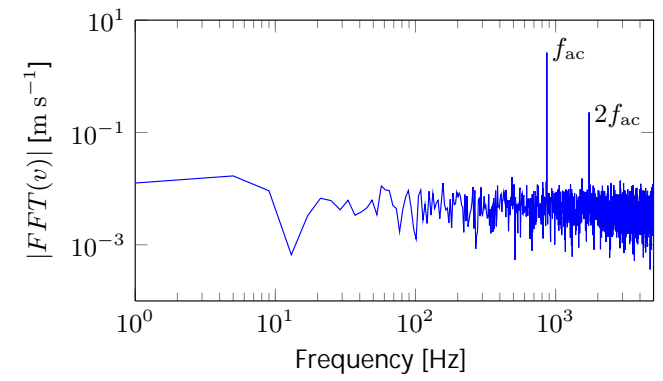

Figure 14. Amplitude spectrum of the flow velocity $v_{y}$ at $(x, y, z)=(0,3.6,0 \mathrm{~mm})$ generated using PIV evaluation.

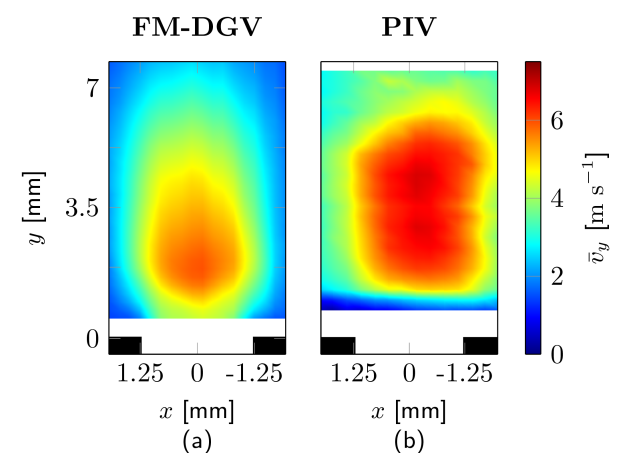

Figure 15. Mean flow velocity $\bar{v}_{y}$ at $z=0 \mathrm{~mm}$ measured with (a) FM-DGV and (b) PIV.

Similar to the validation of the camera-based FM-DGV data in Sect. 3.4, the capability of the PIV evaluation for aeroacoustic investigations needs to be validated. For that reason, the detection of sound-excited flow velocity oscillations is investigated by calculating the averaged spectrum of the velocity $|\mathrm{FFT}(v)|$ at the point $(x, y, z)=(0,3.6,0 \mathrm{~mm})$. This spectrum is shown in Fig. 14. In accordance with the FM-DGV data, the spectrum exhibits the characteristic peaks at $f_{\text {ac }}=867 \mathrm{~Hz}$ and $2 f_{\text {ac }}$. As a result, the capability of the PIV evaluation to resolve sound-excited flow oscillations is proved. However, it is not yet possible to resolve the third or higher harmonics of $f_{\text {ac }}$ as well as the flow turbulence. This is due to the velocity standard deviation of $40 \mathrm{~mm} \mathrm{~s}^{-1}$, which is almost 30 times larger than the standard deviation of the FMDGV at the investigated measurement point. Note that the uncertainties given here are calculated for two-component velocity data. The larger uncertainty of the PIV system is based on the described interrogation setup, which is optimized to correlate the large particle displacements caused by the mean flow velocity. Hence, the weak velocity gradients of the turbulence cannot be resolved (Keane and Adrian, 1990) properly. In contrast, the mean flow velocity can be resolved, which is depicted in Fig. 15. Here a comparison of the mean flow velocity $\bar{v}_{y}$ obtained from the FM-DGV measurement (a) and from the PIV evaluation (b) is presented. The results differ in their shape and positioning relative to the orifice. 
Only the maximum flow velocities $v_{y}=7.1 \mathrm{~m} \mathrm{~s}^{-1}$ due the FM-DGV evaluation and $v_{y}=7.2 \mathrm{~ms}^{-1}$ due the PIV evaluation are in the same range.

The main reason for the disturbance of the PIV evaluation is considered to be the high seeding particle concentration, which causes a low image contrast and makes correlatable structures barely detectable (see Fig. 4). However, also the out-of-plane movement of particles is problematic, when particles enter or leave the measurement volume during the time of $50 \mu$ s between two correlated images (Keane and Adrian, 1990; Nobach and Bodenschatz, 2009). The displacement of particles in $z$ direction is $175 \mu \mathrm{m}$ due to the velocity $v_{z}=3.5 \mathrm{~m} \mathrm{~s}^{-1}$, and thus the displacement is more than half of the light-sheet thickness. Hence, it is most likely that particles occur only in one of the two subsequent images, which leads to a biased estimation of the velocity. Because of the pending issues, the combination of both evaluation techniques yields an increasing uncertainty for all measured velocity components caused by an error propagation. Since two velocity components measured by PIV and one component measured by FM-DGV are combined, the resulting uncertainty calculates to $\left(\sigma_{x}, \sigma_{y}, \sigma_{z}\right)=(40,40,41) \mathrm{mm} \mathrm{s}^{-1}$ based on a coordinate transformation (Schlüßler et al., 2015).

Consequently, the results of the combined FM-DGV-PIV evaluation are not considered further. The used PIV setup and evaluation parameters need to be optimized in order to perform simultaneous three-component measurements. For instance, the reduction of the seeding particle concentration as well as an enhancement of the light-sheet thickness would improve the quality of the PIV measurements. However, both approaches would yield a higher measurement uncertainty for the FM-DGV data due to light intensity fluctuations and a lower signal-to-noise ratio, respectively. As a third approach the additional use of fluorescent seeding particles is possible. This approach would yield better PIV results without an impact on the FM-DGV results, but it would increase the complexity of the measurement system as well. In summary, the combined FM-DGV-PIV requires further investigation, which is valuable due to the possibility of simultaneous highspeed 2-D three-component measurements using only one camera.

\section{Conclusions}

The applicability of the high-speed camera-based FM-DGV system for aeroacoustic investigations is successfully proven. The use of a high measurement rate of $10 \mathrm{kHz}$ and a spatial resolution of $224 \times 224 \times 300 \mu^{3}$ allows the detection of small-scale velocity oscillations in a frequency range up to $5 \mathrm{kHz}$ and down to a power density of $40.5\left(\mathrm{~mm} \mathrm{~s}^{-1}\right)^{2} \mathrm{~Hz}^{-1}$. Furthermore the measurement of two velocity components in a 3-D region of interest $\left(7.2 \times 7.2 \times 3.6 \mathrm{~mm}^{3}\right)$ offers investigations of the maximum mean flow velocity of $7 \mathrm{~m} \mathrm{~s}^{-1}$ and the sound-flow interaction. By performing measurements with this system at a bias flow liner test rig, the detection of soundexcited flow oscillations and of flow turbulence is achieved. Due to this, the periodic (sound-excited flow oscillations) and aperiodic (flow turbulence) flow velocity fluctuations are examined; thus, the energy transfer of the sound into the flow, which contributes to the damping effect of the liner, is shown. Hence, the temporal as well as the spatial behaviour of the flow is investigated.

Additionally an approach for simultaneous threecomponent measurement is shown using an additional PIV evaluation. However, due to the high seeding particle density the results of the PIV evaluation have high uncertainty, because the setup for the measurement using FM-DGV and PIV in combination needs to be improved.

Acknowledgements. The authors thank the German Research Foundation (DFG) for funding the projects

CZ 55/25-3 and EN 797/2-3. Many thanks go to André Döring.

Edited by: M. J. da Silva

Reviewed by: two anonymous referees

\section{References}

Adrian, R. J.: Twenty years of particle image velocimetry, Exp. Fluids, 39, 159-169, 2005.

Bechert, D. W.: Sound absorption caused by vorticity shedding, demonstrated with a jet flow, J. Sound Vib., 70, 389-405, 1980.

Eldredge, J. D. and Dowling, A. P.: The absorption of axial acoustic waves by a perforated liner with bias flow, J. Fluid Mech., 485, 307-335, 2003.

Fischer, A., Büttner, L., Czarske, J., Eggert, M., Grosche, G., and Müller, H.: Investigation of time-resolved single detector Doppler global velocimetry using sinusoidal laser frequency modulation, Meas. Sci. Technol., 18, 2529-2545, 2007.

Fischer, A., König, J., and Czarske, J.: Speckle noise influence on measuring turbulence spectra using time-resolved Doppler global velocimetry with laser frequency modulation, Meas. Sci. Technol., 19, 125402, doi:10.1088/0957-0233/19/12/125402, 2008.

Fischer, A., König, J., Czarske, J., Peterleithner, J., Woisetschläger, J., and Leitgeb, T.: Analysis of flow and density oscillations in a swirl-stabilized flame employing highly resolving optical measurement techniques, Exp. Fluids, 54, 1622, doi:10.1007/s00348013-1622-3, 2013a.

Fischer, A., König, J., Czarske, J., Rakenius, C., Schmid, G., and Schiffer, H. P.: Investigation of the tip leakage flow at turbine rotor blades with squealer cavity, Exp. Fluids, 54, 1462, doi:10.1007/s00348-013-1462-1, 2013b.

Fischer, A., König, J., Haufe, D., Schlüßler, R., Büttner, L., and Czarske, J.: Optical multi-point measurements of the acoustic particle velocity with frequency modulated Doppler global velocimetry, J. Acoust. Soc. Am., 134, 1102-1111, 2013c.

Fischer, A., Schlüßler, R., Haufe, D., and Czarske, J.: Lock-in spectroscopy employing a high-speed camera and a micro-scanner for volumetric investigations of unsteady flows, Opt. Lett., 39, 5082-5085, 2014. 
Hann, D. B. and Greated, C. A.: The measurement of flow velocity and acoustic particle velocity using particle-image velocimetry, Meas. Sci. Technol., 8, 1517-1522, 1997.

Hann, D. B. and Greated, C. A.: The Measurement of Sound Fields Using laser Doppler Anemometry, Acta Acust., 85, 401-411, 1999.

Haufe, D., Schlüßler, R., Fischer, A., Büttner, L., and Czarske, J.: Optical multi-point measurement of the acoustic particle velocity in a superposed flow using a spectroscopic laser technique, Meas. Sci. Technol., 23, 085306, doi:10.1088/0957-0233/23/8/085306, 2012.

Haufe, D., Fischer, A., Czarske, J., Schulz, A., Bake, F., and Enghardt, L.: Multi-scale measurement of acoustic particle velocity and flow velocity for liner investigations, Exp. Fluids, 54, 1569, doi:10.1007/s00348-013-1569-4, 2013.

Haufe, D., Pietzonka, S., Schulz, A., Bake, F., Enghardt, L., Czarske, J., and Fischer, A.: Aeroacoustic near-field measurements with microscale resolution, Meas. Sci. Technol., 25, 105301, doi:10.1088/0957-0233/25/10/105301, 2014a.

Haufe, D., Schulz, A., Bake, F., Enghardt, L., Czarske, J., and Fischer, A.: Spectral analysis of the flow sound interaction at a bias flow liner, Appl. Acoust., 81, 47-49, 2014b.

Henning, A., Kröber, S., and Koop, L.: Acoustic particle velocity measurements: a cross comparison between modern sensor technologies, Meas. Sci. Technol., 24, 085303, doi:10.1088/09570233/24/8/085303, 2013.

Heuwinkel, C., Piot, E., Micheli, F., Fischer, A., Enghardt, L., Bake, F., and Röhle, I.: Characterization of a Perforated Liner by Acoustic and Optical Measurements, in: 16th AIAA/CEAS Aeroacoustics Conf., 7 June-9 June 2010, Stockholm, Sweden, p. 3765,15 pp., 2010.

Keane, R. D. and Adrian, R. J.: Optimization of particle image veIocimeters . Part I : Double pulsed systems, Meas. Sci. Technol., 1, 1202-1215, 1990.

Meier, A. H. and Roesgen, T.: Imaging laser Doppler velocimetry, Exp. Fluids, 52, 1017-1026, 2012.

Müller, H., Eggert, M., Czarske, J., Büttner, L., and Fischer, A.: Single-camera Doppler global velocimetry based on frequency modulation techniques, Exp. Fluids, 43, 223-232, 2007.

Nobach, H. and Bodenschatz, E.: Limitations of accuracy in PIV due to individual variations of particle image intensities, Exp. Fluids, 47, 27-38, 2009.

Petronetto, F., Paiva, A., Lage, M., and Lewiner, T.: Meshless Helmholtz-Hodge Decomposition, IEEE Trans. Vis. Comput. Graph., 16, 338-349, 2010.
Pope, S. B.: Turbulent flows, Cambridge University Press, ISBN: 0521-59125-2, 2000.

Rupp, J., Carrotte, J., and Spencer, A.: Interaction Between the Acoustic Pressure Fluctuations and the Unsteady Flow Field Through Circular Holes, J. Eng. Gas Turbines Power, 132, 061501, doi:10.1115/1.4000114, 2010.

Schlüßler, R., Bermuske, M., Czarske, J., and Fischer, A.: Simultaneous three-component velocity measurements in a swirlstabilized flame, Exp. Fluids, 56, 183, doi:10.1007/s00348-0152055-y, 2015.

Schulz, A., Fischer, A., and Bake, F.: Measurement of the acoustic particle velocity under grazing flow using, in: 17th Int. Symp. Appl. Laser Tech. to Fluid Mech., 1-9, Lisbon, Portugal, 2014.

Schulz, A., Haufe, D., Czarske, J., Fischer, A., Bake, F., and Enghardt, L.: Spectral Analysis of Velocity Fluctuations in the Vicinity of a Bias Flow Liner With Respect to the Damping Efficiency, Acta Acust. united with Acust., 101, 24-36, 2015.

Taylor, K. J.: Absolute measurement of acoustic particle velocity, J. Acoust. Soc. Am., 59, 691-694, 1976.

Thompson, M. W. and Atchley, A. A.: Simultaneous measurement of acoustic and streaming velocities in a standing wave using laser Doppler anemometry, J. Acoust. Soc. Am., 117, 18281838, 2005.

Thurow: MHz Rate Planar Doppler Velocimetry in Supersonic Jets, in: 42nd AIAA Aerosp. Sci. Meet. Exhib., 17 pp., doi:10.2514/6.2004-23, Reno, 2004.

Voigt, A., Bayer, C., Shirai, K., Büttner, L., and Czarske, J.: Laser Doppler field sensor for high resolution flow velocity imaging without camera., Appl. Opt., 47, 5028-5040, 2008.

Watmuff, J. H.: An investigation of the constant-temperature hotwire anemometer, Exp. Therm. Fluid Sci., 11, 117-134, 1995.

Wernet, M. P.: Planar particle imaging Doppler velocimetry: a hybrid PIV/DGV technique for three-component velocity measurements, Meas. Sci. Technol., 15, 2011, 18 pp., 2004.

Willert, C. E., Hassa, C., Stockhausen, G., Jarius, M., Voges, M., and Klinner, J.: Combined PIV and DGV applied to a pressurized gas turbine combustion facility, Meas. Sci. Technol., 17, 16701679, 2006.

Zhao, D., Ang, L., and Ji, C.: Numerical and experimental investigation of the acoustic damping effect of single-layer perforated liners with joint bias-grazing flow, J. Sound Vib., 342, 152-167, 2015. 